\title{
Retraction Note to: Construction of pancreatic cancer double-factor regulatory network based on chip data on the transcriptional level
}

\author{
Li-Li Zhao' ${ }^{1}$ Tong Zhang ${ }^{2}$ - Bing-Rong Liu ${ }^{3}$ Tie-Fu Liu ${ }^{1}$ Na Tao $^{1}$ • \\ Li-Wei Zhuang ${ }^{1}$
}

Published online: 18 August 2015

(C) Springer Science+Business Media Dordrecht 2015

\section{Retraction Note to: Mol Biol Rep (2014) 41:2875-2883 \\ DOI 10.1007/s11033-014-3143-4}

The Publisher and Editor retract this article in accordance with the recommendations of the Committee on Publica tion Ethics (COPE). After a thorough investigation we have strong reason to believe that the peer review process was compromised.

The online version of the original article can be found under doi:10.1007/s11033-014-3143-4.

Li-Wei Zhuang

liweizhuang001@ hotmail.com

1 Department of Gastroenterology, The Fourth Affiliated Hospital of Harbin Medical University, No. 37 Yiyuan Street, Nangang District, Harbin 150001, Heilongjiang, China

2 Department of Cardiology, The Fourth Affiliated Hospital of Harbin Medical University, No. 37 Yiyuan Street, Nangang District, Harbin 150001, Heilongjiang, China

3 Department of Gastroenterology, The Second Affiliated Hospital of Harbin Medical University, No. 148 Baojian Street, Nangang District, Harbin 150001, Heilongjiang, China 